\title{
The Dual Role of the Plastid Terminal Oxidase PTOX: Between a Protective and a Pro-oxidant Function
}

\author{
Anja Krieger-Liszkay * and Kathleen Feilke \\ Institute for Integrative Biology of the Cell, Centre National de la Recherche Scientifique, Comissariat à I'Energie Atomique et \\ aux Energies Alternatives Saclay, Institut de Biologie et de Technologie de Saclay, Université Paris-Sud, Gif-sur-Yvette, France
}

Keywords: plastid terminal oxidase, reactive oxygen species, abiotic stress, photosynthetic electron transport, regulation

The plastid terminal oxidase (PTOX) is a non-heme diiron quinol oxidase that oxidizes plastoquinol and reduced $\mathrm{O}_{2}$ to $\mathrm{H}_{2} \mathrm{O}$. PTOX was discovered in the so-called immutans mutant of $A$. thaliana showing a variegated phenotype (Wetzel et al., 1994; Carol et al., 1999). PTOX is localized in the non-appressed regions of the thylakoid membrane (Lennon et al., 2003) and is involved in carotenoid biosynthesis, plastid development, and chlororespiration. Reviews have focused on the role of PTOX in chlororespiration (Bennoun, 2002; Rumeau et al., 2007), in chloroplast biogenesis (Putarjunan et al., 2013) and in stress responses (McDonald et al., 2011; Sun and Wen, 2011). A recent review by Nawrocki et al. (2015) has addressed the role of PTOX in poising the chloroplast redox potential in darkness. However, its role and interplay with the photosynthetic electron flow remains unclear.

Plants grown in moderate light under non-stress conditions have low PTOX concentrations (about 1 PTOX protein per 100 PSII; Lennon et al., 2003). By contrast, elevated PTOX levels have been found in plants exposed to abiotic stresses such as high temperatures, high light and drought (Quiles, 2006), salinity (Stepien and Johnson, 2009), low temperatures and high intensities of visible (Ivanov et al., 2012), and UV light (Laureau et al., 2013). PTOX has been proposed to act as a safety valve by protecting the plastoquinone pool from overreduction under abiotic stress. A highly reduced PQ pool hinders forward electron flow and triggers charge recombination in photosystem II (PSII) leading to the generation of triplet chlorophyll and highly toxic singlet oxygen. However, overexpression of PTOX in A. thaliana did not protect against light-induced photodamage (Rosso et al., 2006) and even enhanced photo-oxidative stress in tobacco expressing, in addition to its endogenous enzyme, either PTOX from A. thaliana (Heyno et al., 2009) or PTOX1 from C. reinhardtii (Ahmad et al., 2012). Different to higher plants C. reinhardtii possesses two isoforms, PTOX1 and PTOX2. PTOX1 is most likely responsible for regenerating PQ for phytoene desaturation and shows a lower rate of plastoquinol oxidation during photosynthesis than PTOX2 (Houille-Vernes et al., 2011).

Using purified PTOX, Yu and coworkers have recently shown that depending on the quinol concentration PTOX can act as an anti-oxidant or pro-oxidant (Feilke et al., 2014; Yu et al., 2014). PTOX activity was found to be $\mathrm{pH}$ insensitive between $\mathrm{pH}$ 6.0-8.5 when as substrate decylPQH dissolved in methanol was used (Yu et al., 2014). During the catalysis, peroxide intermediates are formed at the diiron center. Depending on the lifetime of these intermediates, reactive oxygen species (ROS) can be generated as a side reaction. Isolated PTOX generates superoxide radicals at both high, but physiologically relevant, quinol concentrations at $\mathrm{pH} 8.0$ and substrate limiting concentrations at pH 6.0-6.5 (Feilke et al., 2014; Yu et al., 2014). When substrate is limited, the second quinol may not arrive in time leading to superoxide formation directly at the catalytic center. Alternatively, since at $\mathrm{pH} 8.0$ the semiquinone is more stable than at $\mathrm{pH} 6.0$, it is conceivable that the high $\mathrm{pH}$ stabilized semiquinone acts as a ROS generator. PTOX in overexpressors has 

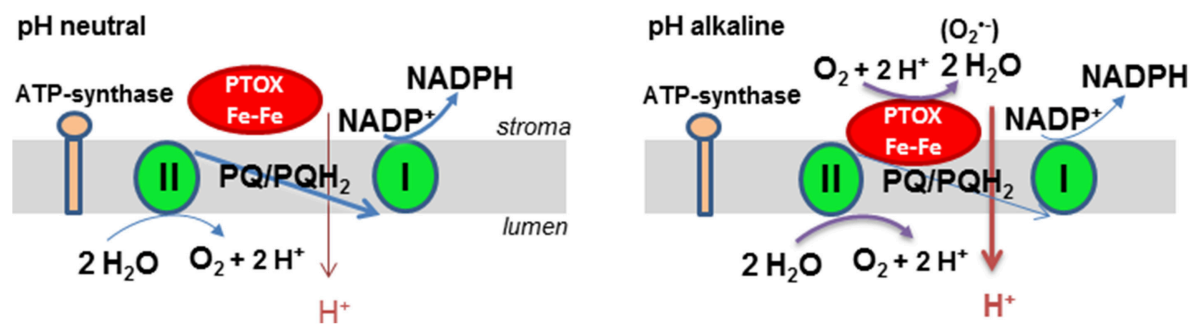

FIGURE 1 | Hypothetical model of the regulation of PTOX activity by the proton gradient in higher plants. Under non-saturating light conditions linear electron transport between PSII and PSI takes place and a moderate proton gradient is established across the thylakoid membrane. PTOX cannot operate since it has no access to its substrate plastoquinol. At saturating light intensities linear electron transport is slowed down, the proton gradient and the plastoquinol concentration increases. The stroma gets more alkaline allowing PTOX to associate to the membrane and to catalyze the oxidation of plastoquinol. Production of $\mathrm{O}_{2}{ }^{\bullet-}$ in a side reaction may trigger a ROS signaling pathway and thereby a stress acclimation response.

also been found to generate superoxide in the light (Heyno et al., 2009).

By oxidizing plastoquinol PTOX reduces the number of electrons available for photosynthetic electron flow. It is generally accepted that PTOX has low activity compared to photosynthetic electron flow. The maximum rate of PTOX was reported to be $5 \mathrm{e}^{-} \mathrm{s}^{-1} \mathrm{PSII}^{-1}$ for PTOX2 in C. reinhardtii and $0.3 \mathrm{e}^{-}$ $\mathrm{s}^{-1} \mathrm{PSII}^{-1}$ in tomato while the maximal rate of photosynthesis is approximately $150 \mathrm{e}^{-} \mathrm{s}^{-1} \mathrm{PSII}^{-1}$ (Nawrocki et al., 2015). However in plants exposed to stress, PTOX activity can account for $30 \%$ of the PSII activity (Stepien and Johnson, 2009). The in vitro enzyme activity of PTOX is high when substrate concentrations are saturating (up to $19.01 \pm 1.1 \mu \mathrm{mol} \mathrm{O}_{2} \mathrm{mg}$ protein $^{-1} \mathrm{~min}^{-1}$; Yu et al., 2014). This corresponds to a turnover rate of $320 \mathrm{e}^{-} \mathrm{s}^{-1} \mathrm{PTOX}^{-1}$ at $35^{\circ} \mathrm{C}$, the optimum temperature for PTOX from rice. The discrepancy between the reported PTOX activities in planta and the $\mathrm{V}_{\max }$ measured with the purified protein points to a mechanism that allows the regulation of PTOX activity depending on the reduction state of the electron transport chain.

Since PTOX can compete with linear and cyclic electron flow (Feilke et al., 2015) and consequently lowers NADPH, ATP production and $\mathrm{CO}_{2}$ fixation and potentially generates ROS, its activity must be tightly controlled. High activity is beneficial for the plant to protect the photosynthetic apparatus against photoinhibition when the electron transport chain is in a highly reduced state as it is the case under abiotic stress when the stomata are closed due to water stress or when $\mathrm{CO}_{2}$ fixation is limited by unfavorable temperatures. However, high PTOX activity is detrimental to high photosynthetic activity when light and $\mathrm{CO}_{2}$ are not limiting.

These observations have led us to postulate the following hypothesis (Figure 1) that explains the discrepancies in the literature about the safety valve function of PTOX. When stromal $\mathrm{pH}$ is alkaline (in high light), PTOX may become associated with the membrane giving it access to its substrate, lipophilic plastoquinol, leading to efficient oxidation of the quinol and reduction of $\mathrm{O}_{2}$ to $\mathrm{H}_{2} \mathrm{O}$. By contrast when stroma $\mathrm{pH}$ becomes less alkaline (under non-saturating light conditions) PTOX may be soluble. Soluble PTOX cannot access its substrate plastoquinol that is located in the thylakoid membrane and the enzyme is effectively inactive. Activity of carotenoid biosynthesis enzymes may be regulated in a similar manner. Phytoene desaturase, which catalyzes the reaction of lipophilic phytoene to $\zeta$-carotene, is found in the stroma both as a tetrameric membrane-bound form which has access to substrate and a soluble multioligomeric form in the stroma that does not (Gemmecker et al., 2015). Another example of an enzyme known to associate with the membrane in a $\mathrm{pH}$-dependent manner is the violaxanthin de-epoxidase (Hager and Holocher, 1994). This enzyme associates with the thylakoid membrane when the luminal $\mathrm{pH}$ decreases.

The model of $\mathrm{pH}$-dependent regulation of PTOX activity by membrane association allows us to rationalize how PTOX could act as a safety valve under conditions of stress such as drought, high light and extreme temperatures when the stomata are closed and the $\mathrm{CO}_{2}$ assimilation rate is low and the stromal $\mathrm{pH}$ is alkaline. Its dissociation from the membrane at less alkaline $\mathrm{pH}$ would hinder its competition with the photosynthetic electron chain for its substrate plastoquinol. Chlororespiration in the dark requires membrane associated PTOX. In our model, this can only take place when a proton gradient is created in the dark by hydrolysis of ATP that is either present in the chloroplast or delivered to the chloroplast from mitochondria. Additionally, when the plastoquinone pool is highly reduced, PTOX can generate superoxide, a potential signaling mechanism that causes the expression levels of responsive genes to change allowing the plant to acclimate to changes in its environment.

\section{ACKNOWLEDGMENTS}

We thank S. Un, CEA Saclay, for critical reading of the manuscript. We thank the CNRS and University Paris-Sud for financial support. 


\section{REFERENCES}

Ahmad, N., Michoux, F., and Nixon, P. J. (2012). Investigating the production of foreign membrane proteins in tobacco chloroplasts: expression of an algal plastid terminal oxidase. PLOS ONE 7:e41722. doi: 10.1371/journal.pone.0041722

Bennoun, P. (2002). The present model for chlororespiration. Photosyn. Res. 73, 273-277. doi: 10.1023/A:1020479920622

Carol, P., Stevenson, D., Bisanz, C., Breitenbach, J., Sandmann, G., Mache, R., et al. (1999). Mutations in the Arabidopsis gene IMMUTANS cause a variegated phenotype by inactivating a chloroplast terminal oxidase associated with phytoene desaturation. Plant Cell 11, 57-68. doi: 10.1105/tpc.11.1.57

Feilke, K., Streb, P., Gabriel, C., Perreau, F., Kruk, J., and Krieger-Liszkay, A. (2015). Effect of Chamydomonas plastid terminal oxidase 1 expressed in tobacco on photosynthetic electron transfer. Plant J. doi: 10.1111/tpj.13101. [Epub ahead of print].

Feilke, K., Yu, Q., Beyer, P., Sétif, P., and Krieger-Liszkay, A. (2014). In vitro analysis of the plastid terminal oxidase in photosynthetic electron transport. Biochim. Biophys. Acta 1837, 1684-1690. doi: 10.1016/j.bbabio.2014.07.016

Gemmecker, S., Schaub, P., Koschmieder, J., Brausemann, A., Drepper, F., Rodriguez-Franco, M., et al. (2015). Phytoene Desaturase from Oryza sativa: Oligomeric Assembly, Membrane Association and Preliminary 3d-Analysis. PLoS ONE 10:e0131717. doi: 10.1371/journal.pone.0131717

Hager, A., and Holocher, K. (1994). Localization of the xanthophyll-cycle enzyme violaxanthin de-epoxidase within the thylakoid lumen and abolition of its mobility by a (light-dependent) $\mathrm{pH}$ decrease. Planta 192, 581-589. doi: 10.1007/BF00203597

Heyno, E., Gross, C. M., Laureau, C., Culcasi, M., Pietri, S., and KriegerLiszkay, A. (2009). Plastid alternative oxidase (PTOX) promotes oxidative stress when overexpressed in tobacco. J. Biol. Chem. 284, 31174-31180. doi: 10.1074/jbc.M109.021667

Houille-Vernes, L., Rappaport, F., Wollman, F.-A., Alric, J., and Johnson, X. (2011). Plastid terminal oxidase 2 (PTOX2) is the major oxidase involved in chlororespiration in Chlamydomonas. Proc. Natl. Acad. Sci. U.S.A. 108, 20820-20825. doi: 10.1073/pnas.1110518109

Ivanov, A. G., Rosso, D., Savitch, L. V., Stachula, P., Rosembert, M., Oquist, G., et al. (2012). Implications of alternative electron sinks in increased resistance of PSII and PSI photochemistry to high light stress in cold-acclimated Arabidopsis thaliana. Photosyn. Res. 113, 191-206. doi: 10.1007/s11120-012-9769-y

Laureau, C., De Paepe, R., Latouche, G., Moreno-Chacón, M., Finazzi, G., Kuntz, M., et al. (2013). Plastid terminal oxidase (PTOX) has the potential to act as a safety valve for excess excitation energy in the alpine plant species Ranunculus glacialis L. Plant Cell Environ. 36, 1296-1310. doi: 10.1111/pce.12059

Lennon, A. M., Prommeenate, P., and Nixon, P. J. (2003). Location, expression and orientation of the putative chlororespiratory enzymes, ndh and immutans, in higher-plant plastids. Planta 218, 254-260. doi: 10.1007/s00425-003-1111-7
McDonald, A. E., Ivanov, A. G., Bode, R., Maxwell, D. P., Rodermel, S. R., and Hüner, N. P. (2011). Flexibility in photosynthetic electron transport: the physiological role of plastoquinol terminaloxidase (PTOX). Biochim. Biophys. Acta 1807, 954-967. doi: 10.1016/j.bbabio.2010.10.024

Nawrocki, W. J., Tourasse, N. J., Taly, A., Rappaport, F., and Wollman, F.-A. (2015). The plastid terminal oxidase: its elusive function points to multiple contributions to plastid physiology. Annu. Rev. Plant Biol. 66, 49-74. doi: 10.1146/annurev-arplant-043014-114744

Putarjunan, A., Liu, X., Nolan, T., Yu, F., and Rodermel, S. (2013). Understanding chloroplast biogenesis using second-site suppressors of immutans and var2. Photosynth. Res. 116, 437-453. doi: 10.1007/s11120-013-9855-9

Quiles, M. J. (2006). Stimulation of chlororespiration by heat and high light intensity in oat plants. Plant Cell Environ. 29, 1463-1470 doi: 10.1111/j.13653040.2006.01510.x

Rosso, D., Ivanov, A. G., Fu, A., Geisler-Lee, J., Hendrickson, L., Geisler, M., et al. (2006). IMMUTANS does not act as a stress-induced safety valve in the protection of the photosynthetic apparatus of Arabidopsis during steady-state photosynthesis. Plant Physiol. 142, 574-585. doi: 10.1104/pp.106.085886

Rumeau, D., Peltier, G., and Cournac, L. (2007). Chlororespiration and cyclic electron flow around PSI during photosynthesis and plant stress response. Plant Cell Environ. 30, 1041-1051. doi: 10.1111/j.1365-3040.2007.01675.x

Stepien, P., and Johnson, G. N. (2009). Contrasting responses of photosynthesis to salt stress in the glycophyte Arabidopsis and the halophyte thellungiella: role of the plastid terminal oxidase as an alternative electron sink. Plant Physiol. 149, 1154-1165. doi: 10.1104/pp.108.132407

Sun, X., and Wen, T. (2011). Physiological roles of plastid terminal oxidase in plant stress responses. J. Biosci. 36, 951-956. doi: 10.1007/s12038-011-9161-7

Wetzel, C. M., Jiang, C.-Z., Meehan, L. J., Voytas, D. F., and Rodermel, S. R. (1994). Nuclear-organelle interactions: the immutans variegation mutant of Arabidopsis is plastid autonomous and impaired in carotenoid biosynthesis. Plant J. 6, 161-175. doi: 10.1046/j.1365-313X.1994.6020161.x

Yu, Q., Feilke, K., Krieger-Liszkay, A., and Beyer, P. (2014). Functional and molecular characterization of plastid terminal oxidase from rice (Oryza sativa). Biochim. Biophys. Acta 1837, 1284-1292. doi: 10.1016/j.bbabio.2014. 04.007

Conflict of Interest Statement: The authors declare that the research was conducted in the absence of any commercial or financial relationships that could be construed as a potential conflict of interest.

Copyright (C) 2016 Krieger-Liszkay and Feilke. This is an open-access article distributed under the terms of the Creative Commons Attribution License (CC BY). The use, distribution or reproduction in other forums is permitted, provided the original author(s) or licensor are credited and that the original publication in this journal is cited, in accordance with accepted academic practice. No use, distribution or reproduction is permitted which does not comply with these terms. 